PROCEEDINGS OF THE

AMERICAN MATHEMATICAL SOCIETY

Volume 133, Number 4, Pages 1205-1212

S 0002-9939(04)07592-6

Article electronically published on October 18, 2004

\title{
COMPACT HOMOMORPHISMS OF URM ALGEBRAS
}

\author{
F. BEHROUZI AND H. MAHYAR
}

(Communicated by N. Tomczak-Jaegermann)

\begin{abstract}
We show when a homomorphism from a URM algebra into a uniform algebra or into a regular Banach algebra is weakly compact or compact. We prove that every homomorphism from URM algebras into $D^{1}(X)$ is compact. Finally, we characterize the spectra of compact endomorphisms of URM algebras defined on a connected compact Hausdorff space $X$.
\end{abstract}

\section{INTRODUCTION}

Let $A$ and $B$ be Banach algebras with maximal ideal spaces $\mathcal{M}(A)$ and $\mathcal{M}(B)$ respectively. In many cases, for a homomorphism $T: A \rightarrow B$ there exists a map $\varphi: \mathcal{M}(B) \rightarrow \mathcal{M}(A)$ such that $\widehat{T f}=\hat{f} \circ \varphi$ for each $f \in A$, where $\hat{f}$ is the Gelfand transform of $f$. In this case, we say $T$ is induced by $\varphi$. The compact homomorphisms of certain Banach algebras have been studied before. For example, D. W. Swanton in [12] characterized compact composition operators on $H^{\infty}(\mathbb{D})$. Also, compact endomorphisms of $H^{\infty}(\mathbb{D})$ and $H^{\infty}\left(B_{E}\right)$ are investigated; see [1], 6] and [13]. Earlier S. Ohno and J. Wada [11] had found sufficient conditions on uniform algebras $A$ and $B$ to ensure that weakly compact homomorphisms between them are actually compact.

In the first section of this note, we study compact homomorphisms of special Banach algebras called URM algebras (Definition 1.1). These algebras include logmodular algebras. We prove that every weakly compact homomorphism from a URM algebra into a regular Banach algebra is compact. We conclude the first section by showing that every homomorphism from a URM algebra into the continuously differentiable function algebra $D^{1}(X)$ on certain compact plane sets $X$ is compact.

In section 2 we determine the spectrum of a nonzero compact endomorphism of URM algebras. J. F. Feinstein and H. Kamowitz in [5] have shown that when $T$ is a compact endomorphism of $H^{\infty}(\mathbb{D})$ induced by an analytic function $\psi: \mathbb{D} \rightarrow \mathbb{D}$, the derivative of $\psi$ at its fixed point determines the spectrum of $T$. Here we extend this result to the URM algebras.

Received by the editors February 2, 2003 and, in revised form, December 18, 2003

2000 Mathematics Subject Classification. Primary 46J10; Secondary 46J15.

Key words and phrases. Compact and weakly compact homomorphism, Gleason part, analytic structure. 


\section{CompaCtness of homomorphisms of URM ALGEBRAS}

A homomorphism between Banach algebras is a bounded linear multiplicative map. Let $A$ be a uniform algebra. As usual, the pseudohyperbolic distance $\rho(m, n)$ for $m, n \in \mathcal{M}(A)$ is defined by

$$
\rho(m, n)=\sup \{|\hat{f}(m)|: f \in A,\|f\| \leq 1, \hat{f}(n)=0\} .
$$

Let $m \in \mathcal{M}(A)$. The set $P(m)=\{n \in \mathcal{M}(A): \rho(m, n)<1\}$ is called the Gleason part of $m$. A Gleason part is said to be nontrivial if it is not a singleton. The properties of the Gleason parts can be found in [10]. The importance of Gleason parts is due to the following facts:

(i) Every Gleason part in $\mathcal{M}(A)$ is clopen relative to the norm topology of $\mathcal{M}(A)$.

(ii) The elements of $A$ often behave like analytic functions when restricted to a Gleason part [6], 13].

(iii) Every Gleason part is clopen relative to the weak topology of $\mathcal{M}(A)$.

Definition 1.1. A uniform algebra $A$ on a compact Hausdorff space $X$ is called a URM algebra, unique representing measure, if every $m \in \mathcal{M}(A)$ has a unique representing measure on $X$.

The notation of URM algebra is used in 6. It is well known that every logmodular algebra is a URM algebra [10. When $A$ is a URM algebra, the weak and the norm topology coincide on $\mathcal{M}(A)[6]$.

The maximal ideal space of a URM algebra has an interesting property, which is called analytic structure; namely, if $A$ is a URM algebra, then every Gleason part is either trivial or an analytic disc in the sense that there exists a one-to-one continuous map from the open unit disc in $\mathbb{C}$ onto every nontrivial Gleason part for $A$. This result, which is called the Wermer-Hoffman-Lumer Theorem, with some corollaries, has been proved by Ülger [13 and reformulated in [6] as follows:

Theorem 1.2. (Wermer-Hoffman-Lumer Theorem). Let $A$ be a URM algebra and $P$ be a nontrivial Gleason part. Then there exists a bijection $\psi_{P}: P \longrightarrow \mathbb{D}=\{z \in$ $\mathrm{C}:|z|<1\}$ such that:

(i) For every $f \in A, \hat{f} \circ \psi_{P}^{-1}$ is a bounded analytic function on $\mathbb{D}$,

(ii) the map $\psi_{P}$ is a homeomorphism if $P$ is given the norm topology of $A^{*}$ and, furthermore,

(iii) there exists a bounded sequence $\left(\boldsymbol{f}_{n}\right)$ in $A$ such that for every $z \in \mathbb{D}, \widehat{\boldsymbol{f}}_{n} \circ$ $\psi_{P}^{-1}(z) \longrightarrow z$ when $n \rightarrow \infty$.

Here we present a result on URM algebras which is an extension of Theorem 4.9 in [13]. The implication $(c \rightarrow b)$ has already been proved in [6] in a different way.

Theorem 1.3. Let $A$ be a URM algebra and $B$ be a uniform algebra on a compact Hausdorff space $X$. Let $T: A \longrightarrow B$ be a unital homomorphism induced by $\varphi$. Then the following statements are equivalent:

(a) $\varphi(X)$ is a norm compact subset of $\mathcal{M}(A)$.

(b) $T$ is compact.

(c) $T$ is weakly compact. 
Proof. $(a \rightarrow b)$ Since $\varphi(X)$ is a norm compact subset of $\mathcal{M}(A)$ and Gleason parts of $\mathcal{M}(A)$ are clopen in $\mathcal{M}(A)$, there exist parts $P_{1}, P_{2}, \ldots, P_{n}$ such that $\varphi(X) \subseteq$ $\bigcup_{i=1}^{n} P_{i}$. Let $\left(g_{j}\right)$ be a bounded sequence in $A$. For compactness of $T$, it is sufficient to show that $\left(\hat{g}_{j}\right)$ has a convergent subsequence on each $P_{i} \cap \varphi(X), i=1,2, \ldots, n$. This is trivial when $P_{i}$ is a singleton. So we show this fact for any nontrivial Gleason part $P_{i}$. By the Wermer-Hoffman-Lumer Theorem there is a bijection $\psi_{P_{i}}: P_{i} \longrightarrow$ $\mathbb{D}$ such that it is a homeomorphism when $P_{i}$ is given the norm topology and $\widehat{g}_{j} \circ \psi_{P_{i}}^{-1}$ is a bounded analytic function on $\mathbb{D}$. Therefore $\left(\widehat{g}_{j} \circ \psi_{P_{i}}^{-1}\right)$ is a bounded sequence of bounded analytic functions on $\mathbb{D}$, and by Montel's Theorem it has a convergent subsequence, which we call $\left(\widehat{g_{j}} \circ \psi_{p_{i}}^{-1}\right)$ again. The sequence $\left(\widehat{g_{j}} \circ \psi_{p_{i}}^{-1}\right)$ is uniformly convergent on every compact subset of $\mathbb{D}$, especially, it is uniformly convergent on the compact subset $\psi_{p_{i}}\left(\varphi(X) \cap P_{i}\right)$. Hence $\left(\widehat{g_{j}}\right)$ is uniformly convergent on $\varphi(X) \cap P_{i}$.

$(b \rightarrow c)$ Clearly.

$(c \rightarrow a)$ If $T: A \longrightarrow B$ is weakly compact, by [4, VI.7.1] $\varphi: \mathcal{M}(B) \longrightarrow \mathcal{M}(A)$ is weak*-weak continuous. Since $X \subseteq \mathcal{M}(B)$ is weak* compact, then $\varphi(X) \subseteq \mathcal{M}(A)$ is weakly compact. By [6] the weak and the norm topology coincide on $\mathcal{M}(A)$. Hence weak compactness of $\varphi(X)$ implies norm compactness of it.

As an application of Theorem 3, we characterize compact endomorphisms of $H^{\infty}(\mathbb{D})$. This result has already been proved in [6].

Corollary 1.4. Let $T: H^{\infty}(\mathbb{D}) \rightarrow H^{\infty}(\mathbb{D})$ be a homomorphism induced by $\varphi$. Then $T$ is compact if and only if the norm closure of $\varphi(\mathbb{D})$ is a norm compact subset of $\mathcal{M}\left(H^{\infty}(\mathbb{D})\right)$.

Proof. By the Corona Theorem, $\mathbb{D}$ is weak* dense in $\mathcal{M}\left(H^{\infty}(\mathbb{D})\right)$. Since norm closure of $\varphi(\mathbb{D})$ is norm compact, so is $\varphi\left(\mathcal{M}\left(H^{\infty}(\mathbb{D})\right)\right)$. The compactness of $T$ then follows from Theorem 3 . The converse is trivial.

Now it is natural to ask whether Theorem 3 remains valid if the uniform algebra is replaced by a Banach algebra in general. For answering this question we need the next theorem.

Theorem 1.5. Let $A$ be a URM algebra and $B$ be either a regular Banach algebra with unit or a uniform algebra. Let $T: A \rightarrow B$ be a homomorphism induced by a nonconstant function $\varphi$. If $\varphi(\mathcal{M}(B))$ is a norm compact subset of $\mathcal{M}(A)$ and contained in one Gleason part $P$, then $\psi_{P} \circ \varphi \in \hat{B}$.

Proof. By hypothesis $P$ is nontrivial. Let $\left(\boldsymbol{f}_{n}\right)$ be a bounded sequence in $A$ obtained by Theorem 2 corresponding to the Gleason part $P$. By passing to a subsequence, we can assume $\hat{f}_{n} \circ \psi_{p}^{-1}(z) \rightarrow z$ uniformly on every compact subset of $\mathbb{D}$, in particular on the compact subset $\psi_{P}(\varphi(\mathcal{M}(B)))$ of $\mathbb{D}$.

In the case that $B$ is a uniform algebra we have $\widehat{T f_{n}}=\hat{f}_{n} \circ \varphi \rightarrow \psi_{P} \circ \varphi$ uniformly on $\mathcal{M}(B)$. That is, $\psi_{P} \circ \varphi \in \hat{B}$.

In the case that $B$ is regular, we must work harder. For the above sequence $\left(\boldsymbol{f}_{n}\right)$, we also have $\left(\hat{f}_{n} \circ \psi_{P}^{-1}\right)^{\prime}(z) \rightarrow 1$ uniformly on the compact subset $\psi_{P}(\varphi(\mathcal{M}(B)))$ of $\mathbb{D}$. Thus $\left(\hat{\boldsymbol{f}_{m}} \circ \psi_{P}^{-1}\right)^{\prime}(z)$ vanishes nowhere on $\psi_{P}(\varphi(\mathcal{M}(B)))$ for some $m$. Hence $\hat{f_{m}} \circ \psi_{P}^{-1}$ is one-to-one in a neighborhood of each point of $\psi_{P}(\varphi(\mathcal{M}(B)))$. 
Fix $x_{0} \in \mathcal{M}(B)$. The analytic function $\hat{\boldsymbol{f}_{m}} \circ \psi_{P}^{-1}$ is one-to-one in a neighborhood $N_{z_{0}}$ of $z_{0}=\psi_{P}\left(\varphi\left(x_{0}\right)\right) \in \psi_{P}(\varphi(\mathcal{M}(B)))$. Then $\left(\hat{f_{m}} \circ \psi_{P}^{-1}\right)^{-1}$ is analytic on the open set $\hat{\boldsymbol{f}_{m}} \circ \psi_{P}^{-1}\left(N_{z_{0}}\right)$. Set $U_{0}=\left(\psi_{P} \circ \varphi\right)^{-1}\left(N_{z_{0}}\right)$. By norm compactness of $\varphi(\mathcal{M}(B)), \varphi$ is weak ${ }^{*}$-norm continuous and then $\psi_{P} \circ \varphi$ is weak* continuous. Hence $U_{0}$ is a weak* neighborhood of $x_{0}$ in $\mathcal{M}(B)$. For every $x \in U_{0}$, we also have $\left(\hat{\boldsymbol{f}_{m}} \circ \psi_{P}^{-1}\right)^{-1}\left(\widehat{\mathrm{Tf}_{m}}(x)\right)=\psi_{P} \circ \varphi(x)$. Therefore $\psi_{P} \circ \varphi \in \hat{B}$, by 14 Theorem $22.10]$.

Theorem 1.6. Let $A$ be a URM algebra and $B$ be a unital commutative Banach algebra. Let $T: A \rightarrow B$ be a homomorphism induced by $\varphi$. If $\varphi(\mathcal{M}(B))$ is a norm compact subset of $\mathcal{M}(A)$, then there exist Gleason parts $P_{1}, P_{2}, \ldots, P_{k}$ such that $\varphi(\mathcal{M}(B)) \subseteq \bigcup_{i=1}^{k} P_{i}$. In addition, if $B$ is either regular or a uniform algebra, then there exists $g \in B$ such that the spectrum of $g$ is contained in $\mathbb{D}$, and for each nontrivial Gleason part $P_{i},\left.\psi_{P_{i}} \circ \varphi\right|_{X_{P_{i}}}=\left.\hat{g}\right|_{X_{P_{i}}}$, where $X_{P_{i}}=\varphi^{-1}\left(P_{i}\right)$.

Proof. Since Gleason parts are mutually disjoint norm open sets and $\varphi(\mathcal{M}(B))$ is a norm compact subset of $\mathcal{M}(A)$, there exist Gleason parts $P_{1}, P_{2}, \ldots, P_{k}$ of $A$ such that $\varphi(\mathcal{M}(B)) \subseteq \bigcup_{i=1}^{k} P_{i}$.

The norm compactness of $\varphi(\mathcal{M}(B))$ implies that $\varphi$ is weak*-norm continuous. Therefore, the $X_{P_{i}}$ 's are mutually disjoint weak*-clopen subsets of $\mathcal{M}(B)$. We set

$$
\theta=\left.\sum \chi_{X_{P_{i}}} \psi_{P_{i}} \circ \varphi\right|_{X_{P_{i}}}+\frac{1}{2} \sum \chi_{X_{P_{i}}}
$$

where $\chi_{X_{P_{i}}}$ is the characteristic function of $X_{P_{i}}$, the first sum is taken over nontrivial Gleason parts and the other one is taken over trivial Gleason parts. By modifications of the proof of Theorem 5 , one can show that:

In the case that $B$ is a uniform algebra, by using the Shilov Idempotent Theorem, $\theta$ is the Gelfand transform of an element $g \in B$ and $g$ is the desired element.

In the case that $B$ is regular, for each $x_{0} \in \mathcal{M}(B)$ there exist a neighborhood $U_{0}$ of $x_{0}$ in $\mathcal{M}(B)$, an element $b \in B$ and an analytic function $G$ on a neighborhood of $\hat{b}\left(x_{0}\right)$ such that $\theta=G \circ \hat{b}$ on $U_{0}$. Now, Theorem 22.10 in [14] can be applied.

We are now in a position to give an affirmative answer to the previous question by showing that Theorem 3 is true for a regular Banach algebra and for the Banach function algebra $D^{1}(X)$.

Theorem 1.7. Let $A$ be a URM algebra and $B$ be a regular Banach algebra with unit. Let $T: A \longrightarrow B$ be a unital homomorphism induced by $\varphi$. Then the following statements are equivalent:

(a) $\varphi(\mathcal{M}(B))$ is a norm compact subset of $\mathcal{M}(A)$.

(b) $T$ is compact.

(c) $T$ is weakly compact.

Proof. $(a \rightarrow b)$ By Theorem 6, there exist Gleason parts $P_{1}, P_{2}, . ., P_{k}$ of $A$, and $g \in B$ with spectrum contained in $\mathbb{D}$ such that $\varphi(\mathcal{M}(B)) \subseteq \bigcup P_{i}$ and $\left.\hat{g}\right|_{X_{P_{i}}}=$ $\left.\psi_{P_{i}} \circ \varphi\right|_{X_{P_{i}}}$ when $P_{i}$ is nontrivial and $\left.\hat{g}\right|_{X_{P_{i}}}=\frac{1}{2}$ otherwise, where $X_{P_{i}}=\varphi^{-1}\left(P_{i}\right)$, for $i=1,2, \ldots, k$. Since the $X_{P_{i}}$ 's are mutually disjoint closed sets of $\mathcal{M}(B)$, the Shilov Idempotent Theorem guarantees that there are $a_{1}, a_{2}, . ., a_{k} \in B$ such that $\hat{a}_{i}$ is the characteristic function of $X_{P_{i}}$. 
For compactness of $T$, suppose $\left(g_{n}\right)$ is a bounded sequence in $A$. If $P_{i}=\left\{x_{i}\right\}$ is a singleton, $a_{i} T g_{n}=\hat{g_{n}}\left(x_{i}\right) a_{i}$. In this case, clearly $\left(a_{i} T g_{n}\right)$ has a convergent subsequence. If $P_{i}$ is nontrivial, $a_{i} T g_{n}=a_{i} \hat{g_{n}} \circ \psi_{P_{i}}^{-1}(g)$. By the Functional Calculus Theorem $\left(a_{i} T g_{n}\right)$ has a convergent subsequence. Since $\sum_{i=1}^{k} a_{i}=1_{B},\left(T g_{n}\right)$ has a convergent subsequence in $B$.

$(b \rightarrow c)$ and $(c \rightarrow a)$ are similar to the proof of Theorem 3 .

Corollary 1.8. Let $A$ be a URM algebra and $B$ be a regular Banach function algebra on a compact Hausdorff space $X$ with $\|f\|_{X}=\|\hat{f}\|_{\mathcal{M}(B)}$ for every $f \in B$ where $\|f\|_{X}=\sup _{x \in X}|f(x)|$. Let $T: A \rightarrow B$ be a homomorphism induced by $\varphi$. Then $T$ is compact if and only if $\varphi(X)$ is a norm compact subset of $\mathcal{M}(A)$.

Proof. Suppose $T$ is compact. By [4, VI.7.1] $\varphi: \mathcal{M}(B) \rightarrow \mathcal{M}(A)$ is weak*-norm continuous. Since $X \subseteq \mathcal{M}(B)$ is a weak* compact subset, $\varphi(X)$ is norm compact. Conversely, let $\varphi(X)$ be a norm compact subset. Thus the homomorphism $f \mapsto T f$, from $A$ into $C(X)$ induced by $\left.\varphi\right|_{X}$ is compact by Theorem 3. Let $\left(g_{n}\right)$ be a bounded sequence in $A$. Since $\left\|T g_{n}\right\|_{X}=\left\|\widehat{T g_{n}}\right\|_{\mathcal{M}(B)}$, then the map $f \mapsto \widehat{T f}$ is a compact homomorphism from $A$ into $C(\mathcal{M}(B))$ induced by $\varphi$. Therefore, $\varphi(\mathcal{M}(B))$ is norm compact, and hence $T$ is a compact homomorphism by Theorem 7 .

We now show that in Theorem 3 the uniform algebra can be replaced by a non-regular function Banach algebra.

Let $X$ be a perfect compact plane set. A complex-valued function $f$ is called differentiable on $X$ if at each point $z_{0} \in X$,

$$
f^{\prime}\left(z_{0}\right)=\lim \left\{\frac{f(z)-f\left(z_{0}\right)}{z-z_{0}}: z \in X, z \rightarrow z_{0}\right\}
$$

exists.

Let $D^{1}(X)$ be the algebra of all continuously differentiable complex-valued functions on $X$, normed by $\|f\|=\|f\|_{X}+\left\|f^{\prime}\right\|_{X}$. The algebra $D^{1}(X)$ was considered by Dales and Davie [3]. In general, the normed algebra $D^{1}(X)$ is not necessarily complete. However, if the compact plane set $X$ is a finite union of (uniformly) regular sets, then $D^{1}(X)$ is complete. In fact, for a perfect compact plane set $X$, the normed algebra $D^{1}(X)$ is complete if and only if for each $z_{0} \in X$ there exists a constant $C$ such that for every $z \in X$ and $f \in D^{1}(X),\left|f(z)-f\left(z_{0}\right)\right| \leq C\left|z-z_{0}\right|\left(\|f\|_{X}+\left\|f^{\prime}\right\|_{X}\right)$ (see [3] and [7]). Such sets include $[0,1]$ and $\overline{\mathbb{D}}$.

For the next theorem, we call a perfect connected compact plane set $X$ good if $D^{1}(X)$ is complete. In this case, $D^{1}(X)$ is a natural Banach function algebra. We now prove another case of Theorem 3 . In fact, we show that every homomorphism from a URM algebra into $D^{1}(X)$ is compact, which is an extension of Theorems 3 and 4 in [9]. The proof of the next result also shows that it is not necessary for the algebra $B$ in Theorem 5 to be regular or uniform.

Theorem 1.9. Let $A$ be a URM algebra and $X$ be a good set. Then every homomorphism from $A$ into $D^{1}(X)$ is compact.

Proof. Suppose $T: A \rightarrow D^{1}(X)$ is a homomorphism induced by $\varphi$. Since $X$ is connected, there exists a Gleason part $P$ such that $\varphi(X) \subseteq P$. If $P$ is a singleton, $T$ is compact since $T$ has a one-dimensional range. Otherwise we show that $\psi_{P} \circ \varphi(X)$ is a compact subset of $\mathbb{D}$. By Theorem 6 in [2] the inclusion map $D^{1}(X) \rightarrow C(X)$ is compact. Then the homomorphism $f \mapsto T f$ from $A$ into $C(X)$ induced by $\varphi$ 
is compact. Hence $\varphi(X)$ is a norm compact subset of $P \subseteq \mathcal{M}(A)$, by Theorem 3 . So $\psi_{P} \circ \varphi(X)$ is a compact subset of $\mathbb{D}$. Let $\left(\boldsymbol{f}_{n}\right)$ be the sequence in $A$ such that $\hat{f}_{n} \circ \psi_{P}^{-1}(z) \rightarrow z$ for every $z \in \mathbb{D}$. Then for every $z \in \mathbb{D}$ there exists $m \in \mathbb{N}$ such that $\left(\hat{\boldsymbol{f}_{m}} \circ \psi_{P}^{-1}\right)^{\prime}(z) \neq 0$. Since $\hat{\boldsymbol{f}_{m}} \circ \varphi=\widehat{T \boldsymbol{f}_{m}}$ is continuously differentiable, so is $\psi_{P} \circ \varphi$.

For compactness of $T$, let $\left(g_{n}\right)$ be a bounded sequence in $A$. Then there exist a subsequence $\left(g_{n_{k}}\right)$ and $g \in H^{\infty}(\mathbb{D})$ such that $\widehat{g_{n_{k}}} \circ \psi_{p}^{-1} \rightarrow g$ and $\left(\widehat{g_{n_{k}}} \circ \psi_{p}^{-1}\right)^{\prime} \rightarrow g^{\prime}$ uniformly on the compact subset $\psi_{P} \circ \varphi(X)$ of $\mathbb{D}$ by Montel's Theorem. Hence $T g_{n_{k}} \rightarrow g \circ\left(\psi_{P} \circ \varphi\right)$ and $\left(T g_{n_{k}}\right)^{\prime} \rightarrow\left(\psi_{P} \circ \varphi\right)^{\prime} g^{\prime}\left(\psi_{P} \circ \varphi\right)$ uniformly on $X$. This means $T$ is compact.

H. Kamowitz and S. Scheinberg in 9] have constructed an example which shows there exists a uniform algebra $A$ and a homomorphism $T$ of $A$ into $C^{1}([0,1])$, induced by $\varphi$, that is not compact. This example also shows that the condition "URM algebra" in Theorem 7 is necessary. For as we know $C^{1}([0,1])$ is regular and the inclusion map $i: C^{1}([0,1]) \rightarrow C([0,1])$ is a compact homomorphism induced by the identity map $i d:[0,1] \rightarrow[0,1]$. So the norm topology and the weak* topology coincide on $[0,1]$ by [4, VI.7.1]. Then $\varphi\left(\mathcal{M}\left(C^{1}([0,1])\right)\right)$ is a norm compact subset of $\mathcal{M}(A)$. But the homomorphism $T: A \rightarrow C^{1}([0,1]$ is not compact.

\section{Spectra of COMPACT ENDOMORPHISMS OF URM ALGEBRAS}

In order to determine the spectrum $\sigma(T)$ of a nonzero compact endomorphism of URM algebras, we need the following results.

Theorem 2.1. Let $A$ and $B$ be URM algebras and $T: A \rightarrow B$ be a homomorphism induced by $\varphi$. Then for every Gleason part $Q$ of $B$ there exists a Gleason part $P$ of $A$ such that $\varphi(Q) \subseteq P$. Furthermore, if $P$ and $Q$ are nontrivial Gleason parts, $\psi_{P} \circ \varphi \circ \psi_{Q}^{-1}$ is analytic on $\mathbb{D}$.

Proof. If $Q$ is a nontrivial Gleason part of $B$, then $Q$ is homeomorphic to $\mathbb{D}$ when $Q$ is given the norm topology, by the Wermer-Hoffman-Lumer Theorem. Since $\varphi: \mathcal{M}(B) \rightarrow \mathcal{M}(A)$ is norm-norm continuous, $\varphi(Q)$ is a norm connected subset of $\mathcal{M}(A)$. Thus there exists one Gleason part $P$ of $A$ such that $\varphi(Q) \subseteq P$.

Let $P$ and $Q$ be nontrivial. By the Wermer-Hoffman-Lumer Theorem, there exists a bounded sequence $\left(\boldsymbol{f}_{n}\right)$ in $A$ such that ${\widehat{\boldsymbol{f}_{n}}}_{\mathrm{f}} \circ \psi_{P}^{-1}(z) \rightarrow z$ for each $z$ in $\mathbb{D}$. Then $\widehat{f}_{n} \circ \varphi \circ \psi_{Q}^{-1}(z) \rightarrow \psi_{P} \circ \varphi \circ \psi_{Q}^{-1}(z)$. By hypothesis, $\widehat{f_{n}} \circ \varphi=\widehat{T f_{n}}$; hence $\widehat{T f_{n}} \circ \psi_{Q}^{-1}(z) \longrightarrow \psi_{P} \circ \varphi \circ \psi_{Q}^{-1}(z)$. Since $\varphi: \mathcal{M}(B) \longrightarrow \mathcal{M}(A)$ is norm-norm continuous, $\psi_{P} \circ \varphi \circ \psi_{Q}^{-1}: \mathbb{D} \longrightarrow \mathbb{D}$ is continuous and also, it is a pointwise limit of a bounded sequence of analytic functions on $\mathbb{D}$. Therefore $\psi_{P} \circ \varphi \circ \psi_{Q}^{-1}$ is analytic on $\mathbb{D}$.

Combining Theorem 1 and the Shilov Idempotent Theorem we have the following result.

Corollary 2.2. Let $A$ be a URM algebra on a connected compact Hausdorff space $X$. Let $T: A \longrightarrow A$ be a compact endomorphism induced by $\varphi$. Then $\varphi(\mathcal{M}(A))$ is contained in one Gleason part $P$. Furthermore, if $P$ is a nontrivial Gleason part, then $\psi_{P} \circ \varphi \circ \psi_{P}^{-1}$ is an analytic function on $\mathbb{D}$. 
Kamowitz in [8] proved that if $T$ is a nonzero compact endomorphism of a semisimple commutative Banach algebra $A$ induced by $\varphi$, then $\bigcap \varphi_{n}(\mathcal{M}(A))$ is finite, where $\varphi_{n}$ is the $n$th iterate of $\varphi$. Moreover, if $\mathcal{M}(A)$ is connected, $\bigcap \varphi_{n}(\mathcal{M}(A))$ is a singleton. In this case, if $\bigcap \varphi_{n}(\mathcal{M}(A))=\left\{x_{0}\right\}, x_{0}$ is a fixed point for $\varphi$.

Theorem 2.3. Let $A$ be a URM algebra on the connected compact Hausdorff space $X$. Let $T: A \longrightarrow A$ be a compact endomorphism induced by the nonconstant function $\varphi$ and $x_{0}$ be the fixed point of $\varphi$. If $P$ is the Gleason part containing $\varphi(\mathcal{M}(A)), \Phi=\psi_{P} \circ \varphi \circ \psi_{P}^{-1}$ and $z_{0}=\psi_{P}\left(x_{0}\right)$, then

$$
\sigma(T)=\left\{\Phi^{\prime}\left(z_{0}\right)^{n}: n \in \mathbb{N}\right\} \cup\{0,1\} .
$$

Proof. Let $0,1 \neq \lambda \in \sigma(T)$. Since $T$ is compact $\lambda$ is an eigenvalue for $T$. Thus there exists $f \in A$ such that $T f=\lambda f$. Therefore we have

$$
\hat{f} \circ \varphi=\widehat{T f}=\lambda \hat{f},
$$

and hence

$$
\hat{f} \circ \psi_{P}^{-1} \circ\left(\psi_{P} \circ \varphi \circ \psi_{P}^{-1}\right)=\lambda \hat{f} \circ \psi_{P}^{-1} .
$$

If we set $F=\hat{f} \circ \psi_{P}^{-1}$, then $F \circ \Phi=\lambda F$ and $F, \Phi$ are analytic functions on $\mathbb{D}$. Since $\Phi\left(z_{0}\right)=z_{0}$ and $\lambda \neq 1, F\left(z_{0}\right)=0$. Let $n$ be a positive integer number such that for every $0 \leq k<n, F^{(k)}\left(z_{0}\right)=0$ but $F^{(n)}\left(z_{0}\right) \neq 0$. We show that $\lambda=\Phi^{\prime}\left(z_{0}\right)^{n}$. By $n$ times differentiation of $F \circ \Phi=\lambda F$, we have $\Phi^{\prime}\left(z_{0}\right)^{n} F^{(n)}\left(\Phi\left(z_{0}\right)\right)=\lambda F^{(n)}\left(z_{0}\right)$; therefore $\lambda=\Phi^{\prime}\left(z_{0}\right)^{n}$. Then $\sigma(T) \backslash\{0,1\} \subseteq\left\{\Phi^{\prime}\left(z_{0}\right)^{n} \mid n \in \mathbb{N}\right\}$.

Conversely, suppose $\lambda=\Phi^{\prime}\left(z_{0}\right)^{m}$ for some $m \in \mathbb{N}$ is not an eigenvalue of $T$. Then $T-\lambda I$ is onto. Suppose $\left(\boldsymbol{f}_{n}\right)$ is the sequence that $\widehat{\boldsymbol{f}}_{n} \circ \psi_{P}^{-1}(z) \longrightarrow z$ for every $z \in \mathbb{D}$. Then there exists a bounded sequence $\left(g_{n}\right) \in A$ such that for each $n \in \mathbb{N}$, $T\left(g_{n}\right)-\lambda g_{n}=\left(\boldsymbol{f}_{n}-z_{0}\right)^{m}$. Therefore $\widehat{T\left(g_{n}\right)} \circ \psi_{P}^{-1}-\lambda \widehat{g_{n}} \circ \psi_{P}^{-1}=\left(\widehat{\boldsymbol{f}}_{n} \circ \psi_{P}^{-1}-z_{0}\right)^{m}$. If we set $F_{n}=\hat{\boldsymbol{f}}_{n} \circ \psi_{P}^{-1}$ and $G_{n}=\hat{g_{n}} \circ \psi_{P}^{-1}$, we have

$$
G_{n} \circ \Phi-\lambda G_{n}=\left(F_{n}-z_{0}\right)^{m} .
$$

Since $\left(F_{n}\right)$ and $\left(G_{n}\right)$ are bounded sequences of analytic functions on $\mathbb{D}$ and $F_{n}(z)$ $\longrightarrow z$ for each $z \in \mathbb{D}$, by Montel's Theorem there exists an analytic function $G$ on $\mathbb{D}$ such that

$$
G \circ \Phi(z)-\lambda G(z)=\left(z-z_{0}\right)^{m} .
$$

If $\Phi^{\prime}\left(z_{0}\right)=0$ or $\left|\Phi^{\prime}\left(z_{0}\right)\right|=1$, the proof is complete. But if $\Phi^{\prime}\left(z_{0}\right) \neq 0$ and $\left|\Phi^{\prime}\left(z_{0}\right)\right| \neq$ 1 , since $\Phi: \mathbb{D} \rightarrow \mathbb{D}$ is analytic, by $m-1$ times differentiation, we have $G\left(z_{0}\right)=$ $G^{\prime}\left(z_{0}\right)=\cdots=G^{(m-1)}\left(z_{0}\right)=0$, and by $m$ times differentiation, we have $0=$ $\Phi^{\prime}\left(z_{0}\right)^{m} G^{(m)}\left(\Phi\left(z_{0}\right)\right)-\lambda G^{(m)}\left(z_{0}\right)=m$ !, which is a contradiction.

\section{REFERENCES}

[1] R. Aron, P. Galindo and M. Lindström, Compact homomorphisms between algebras of analytic functions, Studia Math. 123 (1997), 235-247. MR98h:46053

[2] F. Behrouzi, Homomorphisms of certain Banach function algebras, Proc. Indian Acad. Sci. (Math. Sci.) 112 (2002), 331-336. MR2003e:46083

[3] H. G. Dales and A. M. Davie, Quasianalytic Banach function algebras, J. Funct. Anal. 13 (1973), 28-50. MR49:7782

[4] N. Dunford and J. T. Schwartz, Linear Operators, Part I, General Theory, Interscience Publ. New York, 1958. MR90g:47001a

[5] J. F. Feinstein and H. Kamowitz, Compact endomorphisms of $H^{\infty}(D)$, Studia Math. 136 (1999), 87-90. MR2001h:46093 
[6] P. Galindo and M. Lindström, Gleason parts and weakly compact homomorphisms between uniform Banach algebras, Monatsh. Math. 128 (1999), 89-97. MF2002a:46069

[7] T. G. Honary and H. Mahyar, Approximation in Lipschitz algebras of infinitely differentiable functions, Bull. Korean Math. Soc. 36 (1999), 629-636. MR2001b:46083

[8] H. Kamowitz, Compact endomorphisms of Banach algebras, Pacific J. Math. 89 (1980), 313325. MR 82c: 46063

[9] H. Kamowitz and S. Scheinberg, Homomorphisms of Banach algebras with range in $C^{1}([0,1])$, Inter. J. Math. 2 (1994), 201-212. MR95b:46073

[10] G. M. Leibowitz, Lectures on Complex Function Algebras, Scott, Foresman and Co., Glenview, Illinois, 1970. MR.55:1072

[11] S. Ohno and J. Wada, Compact homomorphisms on function algebras, Tokyo J. Math. 4 (1981), 105-112. MR82i:46079

[12] D. W. Swanton, Compact composition operators on B(D), Proc. Amer. Math. Soc. 56 (1976), 152-156. MF $53: 11420$

[13] A. Ülger, Some results about the spectrum of commutative Banach algebras under the weak topology and applications, Monatsh. Math. 121 (1996), 353-379. MR98a:46058

[14] W. Zelazko, Banach Algebras, Elsevier Publ. Co, 1973. MR56:6389

Faculty of Mathematical Sciences and Computer Engineering, Teacher Training UniVERSITY, TEHRAN 15618, IRAN

E-mail address: behrouzif@yahoo.com

Faculty of Mathematical Sciences and Computer Engineering, Teacher Training UniVERsity, TeHran 15618, Iran

E-mail address: mahyar@saba.tmu.ac.ir 